\title{
An Overview of Treatment Drugs Affecting Survival of Patients with Acute Lymphoblastic Leukemia
}

\author{
Zerina Đozić \\ Department of Genetics and Bioengineering \\ International Burch University \\ Sarajevo, Bosnia and Herzegovina
}

\begin{abstract}
Acute lymphoblastic leukemia (ALL) is a blood cancer characterized by accumulation of immature white blood cells known as lymphoblasts. Commonly, ALL appears in childhood however, it is also present in adulthood. One of the causes of ALL is BCR-ABL translocation called Philadelphia chromosome which includes chromosome 9 and 22 . The fusion of BCR-ABL results in hybrid protein (tyrosine kinase protein) which causes cells to divide uncontrollably. Consequently, the main role of the treatment drugs is to target this protein and to stop its function. This paper evaluates three different types of targeted treatment drug: imatinib, dasatinib and nilotinib and their effect on patients' survival. Based on overall survival most successful drug for the treatment of patients with acute lymphoblastic leukemia was imatinib $(75 \%)$. Highest median age (69 years) was observed in patients with dasatinib. Targeted cancer therapy is the treatment strategy which can precisely target the basis of the disease and stop its progression. In order for patients to respond best to particular therapy the production of new and improved targeted drugs is unavoidable.
\end{abstract}

Keywords- Acute Lymphoblastic Leukemia, Overall Survival, Philadelphia Chromosome, Translocation.

\section{INTRODUCTION}

Acute lymphoblastic leukemia (ALL) is a hematologic malignancy propagated by impaired differentiation, proliferation, and accumulation of lymphoid progenitor cells in the bone marrow and/or extramedullary sites. Although ALL occurs predominantly in children, it is adult ALL that is more challenging to treat [1].

Originally thought to be associated with chronic myelogenous leukemia (CML) only, Philadelphia translocation has also been identified in acute lymphoblastic leukemia (ALL). The translocation results in a BCR-ABL fusion protein when the protooncogene $\mathrm{ABL}$ moves from chromosome 9 to the major breakpoint cluster region within the BCR gene on chromosome 22 [2].

Philadelphia chromosome-positive $(\mathrm{Ph}+)$ acute lymphoblastic leukemia (ALL) is present in patients for about one quarter of adult cases of ALL [3].

There is a scarcity of reported genomic data for adults because most ALL studies have been performed in pediatric patients. Philadelphia chromosome $(\mathrm{Ph})$-like ALL is a recently described entity with a poor prognosis. It occurs in approximately $10 \%$ of children and up to $30 \%$ of young adults with ALL [4].

Unfortunately, the treatment success of pediatric ALL has not been mimicked in adult ALL. Despite high rates of complete remission (CR) (80\%-90\%) in adult ALL, the cure rates are only $40 \%$ to $50 \%$ because of relapses. The 5-year overall survival (OS) is approximately $90 \%$ in children and $30 \%$ to $40 \%$ in adults and elderly patients. This problem may be attributed to adults harboring higher-risk features at diagnosis, increased comorbidities, and the development of chemotherapy resistance after relapse. The need for improvement in adult ALL outcomes has led to major advancements in drug development, reassessment of risk stratification, and better knowledge of disease pathogenesis. The incorporation of targeted therapies in the frontline and salvage settings has improved survival compared with that of conventional chemotherapy in adult ALL. However, the ultimate goal is to further optimize treatment regimens to achieve success similar to pediatric ALL [5].

\section{EASE OF USE}

The aim of this paper is to compare the current information about treatment drugs used for $\mathrm{Ph}+$ acute lymphoblastic leukemia (ALL).

TABLE I. Treatment drugs affecting patients' survival and papers used for comparison.

\begin{tabular}{|l|c|c|c|}
\hline \multicolumn{4}{|c|}{$\mathrm{Ph}+$ Acute Lymphoblastic Leukemia } \\
\hline Treatment drug & Imatinib & Dasatinib & Nilotinib \\
\hline Article & $\begin{array}{c}\text { D.Thomas } \\
\text { et al. } \\
(2004)\end{array}$ & $\begin{array}{c}\text { R. Foà et } \\
\text { al. } \\
(2011)\end{array}$ & $\begin{array}{c}\text { D. Kim } \\
\text { et al. } \\
(2015)\end{array}$ \\
\hline Article & $\begin{array}{c}\text { Ottmann } \\
\text { O.G. et al. } \\
(2002)\end{array}$ & $\begin{array}{c}\text { R.Philippe } \\
\text { et al. } \\
(2016)\end{array}$ & $\begin{array}{c}\text { Hoffman } \\
\text { J. } \\
(2014)\end{array}$ \\
\hline
\end{tabular}

\section{RESULTS}

Three articles are used for comparison of treatment drugs affecting patients' survival. Below is a brief analysis of each article with a focus on treatment responses in acute lymphoblastic leukemia patients. 


\section{A. $\quad$ D. Thomas et al. (2004) [6]}

The purpose of this study was to use concurrent chemotherapy and imatinib mesylate in newly diagnosed $\mathrm{Ph}$ positive ALL patients. Imatinib mesylate, an inhibitor of the Bcr-Abl tyrosine kinase, has modest activity in refractory/relapsed Philadelphia chromosome (Ph)-positive acute lymphocytic leukemia (ALL). There were 20 patients who received hyper-CVAD (cyclophosphamide, vincristine, Adriamycin, and dexamethasone) and imatinib mesylate followed by imatinib mesylate-based consolidation/maintenance therapy. Of the patients, $11(55 \%)$ presented with de novo disease, $4(20 \%)$ were refractory to standard induction chemotherapy, and 5 (25\%) entered the study in CR after one course of induction chemotherapy. The median age of the group was 42 years (range, 17 to 75 years); $70 \%$ were males.

\section{B. $\quad$ R. Foà et al. (2011) [7]}

This study is about fifty-five adult patients with $\mathrm{Ph}^{+} \mathrm{ALL}$ which were enrolled in the study for dasatinib treatment. Dasatinib is a potent BCR-ABL tyrosine kinase inhibitor effective in chronic myeloid leukemia and $\mathrm{Ph}^{+}$acute lymphoblastic leukemia (ALL) resistant/intolerant to imatinib. Of 53 evaluable patients, 28 were females and 25 males. The median age was 53.6 years (range, 23.8-76.5 years), with 12 patients older than 60 years. All patients achieved a complete hematologic remission (CHR), 49 (92.5\%) at day 22. At this time point, 10 patients achieved a BCR-ABL reduction to < $10^{-3}$. At 20 months, the overall survival was $69.2 \%$ and diseasefree survival was $51.1 \%$.

\section{D. Kim et al. (2015) [8]}

In this study 91 subjects were enrolled in 17 centers in the study for nilotinib plus chemotherapy treatment. The advantages of nilotinib include its high in vitro affinity to BCRABL1 tyrosine kinase. Investigation of the effects of nilotinib plus multiagent chemotherapy was done, followed by consolidation/maintenance or allogeneic hematopoietic cell transplantation (allo-HCT) for adult patients with newly diagnosed Philadelphia-positive (Ph-pos) acute lymphoblastic leukemia (ALL). Moreover, the high incidence of ABL1 kinase mutations in Ph-pos ALL diagnoses justifies the use of nilotinib because nilotinib can effectively inhibit various mutated BCRABL1 tyrosine kinases. Drugs used are vincristine, daunorubicin, prednisolone, and nilotinib. Out of 91 patients $50 \%$ where males and $50 \%$ were females. Median age was 47 years with the range between 17 and 71 years. The overall survival rate was $72 \%$ when survival time was measured to the date of last follow-up or death.

Ottmann O.G. et al. (2002) [9]

In this study is conducted a clinical trial in 56 patients with relapsed or refractory $\mathrm{Ph}+$ acute lymphoblastic leukemia (ALL; 48 patients) or chronic myelogenous leukemia in lymphoid blast crisis (LyBC; 8 patients). Male or female patients were eligible for inclusion in this study if they were at least 18 years of age and had a morphologically confirmed diagnosis of relapsed or refractory $\mathrm{Ph}+\mathrm{ALL}$ or LyBC. Imatinib was given once daily at $400 \mathrm{mg}$ or $600 \mathrm{mg}$. Imatinib induced complete hematologic responses (CHRs) and complete marrow responses
(marrow-CRs) in 29\% of ALL patients (CHR, 19\%; marrowCR, $10 \%$ ), which were sustained for at least 4 weeks in $6 \%$ of patients. Median estimated time to progression and overall survival for ALL patients were 2.2 and 4.9 months, respectively. CHRs were reported for $3(38 \%)$ of the patients with LyBC (one sustained CHR).

\section{R. Philippe et al. (2016) [10]}

This study is about investigation of dasatinib, another potent tyrosine kinase inhibitor, in combination with low-intensity chemotherapy. Patients older than 55 years were treated with dasatinib $140 \mathrm{mg} /$ day (100 mg/day over 70 years) with intrathecal chemotherapy, vincristine and dexamethasone during induction. Patients in complete remission continued consolidation with dasatinib, reduced doses of sequential cytarabine and methotrexate for 6 months. Maintenance therapy was dasatinib and vincristine/dexamethasone re-inductions for 18 months followed by dasatinib until relapse or death. Seventy-one patients with a median age of 69 years were enrolled, $77 \%$ had a high comorbidity score. Only seven patients underwent allogeneic hematopoietic stem cell transplantation. At 5-years, overall survival was $36 \%$ and up to $45 \%$ taking into account deaths unrelated to disease or treatment as competitors. Finally, dasatinib combined with low-intensity chemotherapy was well tolerated and gave long-term survival in $36 \%$ of elderly patients with Ph+ ALL. Monitoring of BCRABL1 from diagnosis identified patients with at high risk of early relapse and may help to switch therapy.

\section{Hoffman J. (2014) [11]}

As of October 2014, researchers had enrolled 56 patients with a median age of 65 years with $\mathrm{Ph}+$ and/or BCR-ABL1positive ALL. Patients first received dexamethasone on days -7 to -3 with or without cyclophosphamide IV on days -3 to -1 in a pre-phase. Then, all patients received nilotinib $400 \mathrm{mg}$ twice daily beginning during induction and continuing thereafter, in addition to vincristine and dexamethasone on 2 days, weekly for 4 weeks. Patients received nilotinib twice daily, 6mercaptopurine daily, and methotrexate once weekly for 4 weeks every other month, and dexamethasone and vincristine in 2-month intervals for up to 2 years. Of those enrolled, 47 were evaluable for efficacy by the time of this analysis. Patients were followed for a median of 8.5 months. The complete hematologic response rate following treatment was $87 \%$ with a median time to complete response of 41 days. In addition, $4 \%$ of patients experienced a partial response or failure, $6 \%$ discontinued treatment before assessment, and $2 \%$ died. At 30 months, the overall survival rates were $72.7 \%$ for patients who did not undergo stem cell transplantation and $67.1 \%$ for all patients.

TABLE II. Comparison of patients' characteristics which were using three different types of treatment for $\mathrm{Ph}+$ acute lymphoblastic leukemia.

\begin{tabular}{|c|c|c|c|}
\hline Treatment drug & Imatinib & Dasatinib & Nilotinib \\
\hline $\begin{array}{c}\text { Number of patients } \\
\text { (first article) }\end{array}$ & 20 & 53 & 91 \\
\hline $\begin{array}{c}\text { Median age (first } \\
\text { article) }\end{array}$ & $\begin{array}{c}42 \\
(17-75)\end{array}$ & $\begin{array}{c}69 \\
(23-76)\end{array}$ & $\begin{array}{c}47 \\
(17-71)\end{array}$ \\
\hline $\begin{array}{c}\text { Number of patients } \\
\text { (second article) }\end{array}$ & 48 & 71 & 56 \\
\hline $\begin{array}{c}\text { Median age (second } \\
\text { article) }\end{array}$ & $\begin{array}{c}46 \\
(18-75)\end{array}$ & $\begin{array}{c}69 \\
(55-79)\end{array}$ & $\begin{array}{c}60 \\
(50-71)\end{array}$ \\
\hline
\end{tabular}




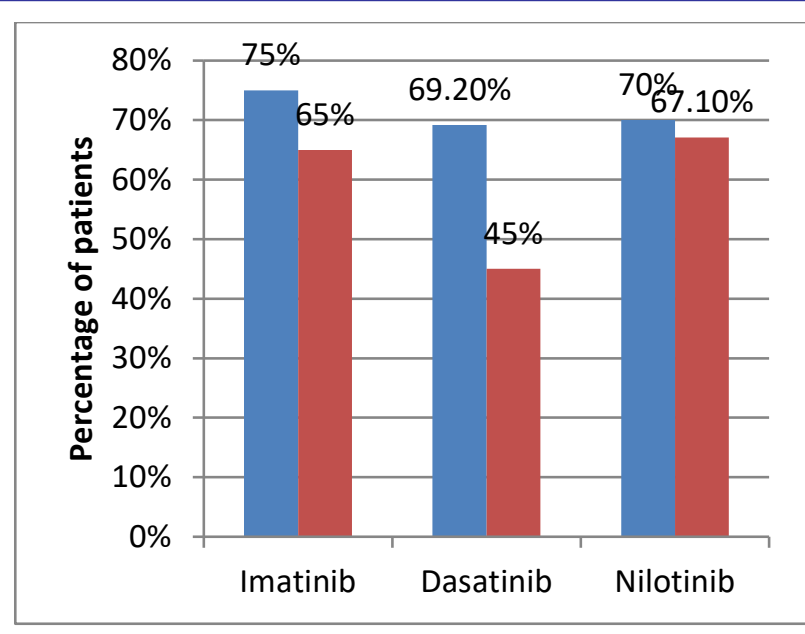

Figure 1. Comparison of overall survival of patients with $\mathrm{Ph}+$ acute lymphoblastic leukemia using different treatment drugs.

\section{DISCUSSION}

Before the era of imatinib, the HCR (hematologic remission) rate for Ph-positive all was $64 \%$ to $83 \%$ with high-dose multiagent chemotherapy, and the median survival time was only $\sim 1$ year. The introduction of imatinib into the treatment of Ph-pos ALL has increased the HCR rate to $95 \%$ to $100 \%$ and has achieved meaningful improvement in OS. Despite its overall benefits, imatinib has some limitations as a treatment of Ph-pos ALL [7]. First, the gastrointestinal AEs of imatinib may be aggravated when combined with cytotoxic drugs; this aggravation results in reduced drug compliance and, consequently, reduced dose intensity. Second, among the patients ineligible for allo-HCT that received consolidation followed by maintenance, many relapsed after discontinuing imatinib. That result suggested that even when imatinib was included in the treatment, allo-HCT remained important for overcoming the poor prognosis of $\mathrm{Ph}$ positive ALL. The advantages of nilotinib include its high in vitro affinity to BCRABL1 tyrosine kinase, its improved MR in the treatment of chronic-phase CML and ALL. In agreement with previous findings with conventional chemotherapy, indicate that patients with $\mathrm{Ph}+\mathrm{ALL}$ have a heterogeneous sensitivity to dasatinib and suggest that a delayed or less profound molecular response during dasatinib induction identifies patients with an increased relapse likelihood. The rates of OS and DFS of $69.2 \%$ and $51.1 \%$ at 20 months of dasatinib study, clearly influenced by the postremission treatment, compare favorably with the data reported in the literature, particularly in view of the overall older age of the patients' series [8].

The survival of the patients depends on the years of the patient, time of the discovering disease, and finally of the type of the treatment and drug used in it. Imatinib, dasatinib and nilotinib are mostly used. All of these three drugs are inhibitors of tyrosine kinase protein. In this paper it is expected to have more significant percentage of survival of the particular drugs. The highest percentage of survival had imatinib (75\%), and the lowest had dasatinib (45\%). Probably, dasatinib had low survival because of the median age of the patients, because they were very olf (69 median age). Finally, this paper could help other researchers who are interested in statistical analysis of $\mathrm{Ph}$ positive lymphoblastic leukemia.

\section{CONCLUSION}

To conclude, this article was made to compare the six clinical trials (two for imatinib mesylate, two for dasatinib and two for nilotinib drug) that were already done and published, and to compare the percentage of the survival of the patients suffered from $\mathrm{Ph}$ positive acute lymphoblastic leukemia. In this article, it has been calculated the overall survival for three different drugs and treatments used in clinical trials. Furthermore, in the comparing it can be concluded that the imatinib mesylate drug had the highest overall survival and other two drugs, dasatinib and nilotinib had almost the same overall survival in the first two articles, but in the second one they differ. The overall survival can also depend on how much patients the trial was done and how old the patients were. For imatinib mesylate, there were 20 and 48 patients in the tested treatment, for dasatinib 53 and 71 patients and for nilotinib 91 and 56 patients were put on the treatment. Finally, there is higher percentage of difference of overall survival of the patients for dasatinib because the second trial was done on elderly patients.

\section{REFERENCES}

[1] De Labarthe, A., et al (2007). Imatinib combined with induction or consolidation chemotherapy in patients with de novo Philadelphia chromosome-positive acute lymphoblastic leukemia: results of the GRAAPH-2003 study. Blood, 109(4), 1408-1413.

[2] Dombret, H., et al (2002). Outcome of treatment in adults with Philadelphia chromosome-positive acute lymphoblastic leukemiaresults of the prospective multicenter LALA-94 trial. Blood, 100(7), 2357-2366.

[3] Adele K. Fielding (2010). Current Treatment of Philadelphia Chromosome-Positive Acute Lymphoblastic Leukemia. Haematologica. 95: 8-12

[4] Paul, Shilpa et al. Adult Acute Lymphoblastic Leukemia. Mayo Clinic Proceedings, Volume 91, Issue 11, 1645 - 1666

[5] A. K. Fielding, G. A. Zakout.Treatment of Philadelphia chromosomepositive acute lymphoblastic leukemia. Curr Hematol Malig Rep. 2013 Jun; 8(2): 98-108.

[6] Thomas, D. et al (2004). Treatment of Philadelphia chromosomepositive acute lymphocytic leukemia with hyper-CVAD and imatinib mesylate. Blood,103(12), 4396-4407.

[7] Foà, R., et al. (2011). Dasatinib as first-line treatment for adult patients with Philadelphia chromosome-positive acute lymphoblastic leukemia. Blood, 118(25), 6521-6528.

[8] Kim, D., et al (2015), Nilotinib combined with multiagent chemotherapy for newly diagnosed Philadelphia-positive acute lymphoblastic leukemia. Blood, 126(6), 746-756.

[9] Ottmann, O. G. et al. (2002). A phase 2 study of imatinib in patients with relapsed or refractory Philadelphia chromosome-positive acute lymphoid leukemias. Blood, 100(6), 1965-1971. Accessed April 20, 2017.

[10] Rousselot, P., Coudé, et al. (2016). Dasatinib and low-intensity chemotherapy in elderly patients with Philadelphia chromosomepositive ALL. Blood, (), blood-2016-02-700153.

[11] Jason Hoffman (2014). Nilotinib Plus Chemotherapy Effective for First-Line Treatment of Acute Lymphoblastic Leukemia (ALL), (2014). Cancer Therapy Advisor. 